OPEN ACCESS

Edited by:

David J. Seiffge,

University Hospital Bern, Switzerland

Reviewed by:

Qi Li,

First Affiliated Hospital of Chongqing Medical University, China Achim Müller, University Hospital Zürich, Switzerland Thien J. Huynh,

University of Florida College of Medicine-Jacksonville, United States

*Correspondence: Xingquan Zhao zxq@vip.163.com

Specialty section: This article was submitted to

Stroke,

a section of the journal

Frontiers in Neurology

Received: 14 February 2020

Accepted: 27 August 2020

Published: 06 October 2020

Citation:

Wang J, Wang W, Liu Y and Zhao $X$ (2020) Associations Between Levels of High-Sensitivity C-Reactive Protein and Outcome After Intracerebral Hemorrhage.

Front. Neurol. 11:535068. doi: 10.3389/fneur.2020.535068

\section{Associations Between Levels of High-Sensitivity C-Reactive Protein and Outcome After Intracerebral Hemorrhage}

\author{
Jing Wang ${ }^{1,2,3,4,5}$, Wenjuan Wang ${ }^{1,2,3,4,5}$, Yanfang Liu ${ }^{1,2,3,4,5}$ and Xingquan Zhao ${ }^{1,2,3,4,5 *}$ \\ ${ }^{1}$ Department of Neurology, Beijing Tiantan Hospital, Capital Medical University, Beijing, China, ${ }^{2}$ China National Clinical \\ Research Center for Neurological Diseases, Beijing, China, ${ }^{3}$ Center of Stroke, Beijing Institute for Brain Disorders, Beijing, \\ China, ${ }^{4}$ Beijing Key Laboratory of Translational Medicine for Cerebrovascular Disease, Beijing, China, ${ }^{5}$ Beijing Key \\ Laboratory of Central Nervous System Injury, Beijing, China
}

Background: Patients with spontaneous intracerebral hemorrhage $(\mathrm{ICH})$ have high mortality and morbidity rates; approximately one-third of patients with $\mathrm{ICH}$ experience hematoma expansion (HE). The spot sign is an established and validated imaging marker for HE. High-sensitivity C-reactive protein (hs-CRP) is an established laboratory marker for inflammation and secondary brain injury following $\mathrm{ICH}$.

Objective: To determine the association between the spot sign and hs-CRP, hematoma expansion, and clinical outcomes.

Methods: Between December 2014 and September 2016, we prospectively recruited 1,964 patients with acute symptomatic $\mathrm{ICH}$ at 13 hospitals in Beijing, China. Next, we selected 92 patients within $24 \mathrm{~h}$ of the onset of symptoms from this cohort for the present study. $\mathrm{ICH}$ was diagnosed in the emergency room by non-contrast computed tomography (NCCT) scans. Follow-up scans were carried out within $48 \mathrm{~h}$ to evaluate patients for HE. Multidetector computed tomography angiography (MDCTA) was also used to identify spot signs. Blood samples were collected from each patient at admission in EDTA tubes (for plasma) or vacutainer tubes (for serum). hs-CRP values were determined by a particle-enhanced immunoturbidimetric assay in the laboratory at Beijing Tiantan Hospital, Capital Medical University. Patients were categorized into two groups according to their hs-CRP levels (hs-CRP $<3 \mathrm{mg} / \mathrm{L}$, hs-CRP $\geq 3 \mathrm{mg} / \mathrm{L}$ ).

Results: The incidences of spot sign and HE in our study cohort were 31.5 and 29.3\%, respectively. Following the removal of potential confounding variables, stepwise-forward logistic regression analysis identified that an $\mathrm{hs}-\mathrm{CRP}$ level $\geq 3 \mathrm{mg} / \mathrm{L}$ was not a significant indicator for either spot sign $(p=0.68)$ or HE $(p=0.07)$. However, an hs-CRP level $\geq 3$ $\mathrm{mg} / \mathrm{L}$ (odds ratio: 16.64, 95\% confidence interval: $2.11-131.45, p=0.008$ ) was identified as an independent predictor of an unfavorable outcome 1 year after acute $\mathrm{ICH}$.

Conclusions: Our analyses identified that an hs-CRP level $\geq 3 \mathrm{mg} / \mathrm{L}$ was a significant indicator for an unfavorable outcome 1 year after acute $\mathrm{ICH}$.

Keywords: spot sign, hematoma expansion, spontaneous intracerebral hemorrhage, clinical outcome, high-sensitivity CRP 


\section{INTRODUCTION}

Spontaneous intracerebral hemorrhage (ICH) is a devastating subtype of stroke with high global rates of mortality and morbidity (1). ICH is responsible for over half of all deaths caused by stroke, and functional independence after ICH is known to range from 12 to $39 \% 1$ year or longer (1). Up to one-third of patients with ICH may experience hematoma expansion (HE), thus leading to a higher mortality rate and unfavorable clinical outcomes $(2,3)$. Therefore, it is vital that we develop methods to prevent and manage $\mathrm{HE}$ in patients experiencing acute ICH. The spot sign is a well-established and validated imaging marker for HE (4). However, identifying the spot sign requires the application of computed tomography angiography (CTA) with the additional injection of contrast materials and exposure to radiation. Furthermore, neuroradiologists are needed for processing and reconstructing images acquired by CTA. Previous randomized controlled trials reported that $<25 \%$ of patients with ICH underwent CTA during the acute phase $(5,6)$. Other research studies have reported that a number of indicators are associated with HE and poor outcomes in patients with ICH, including non-contrast CT (NCCT) markers, such as computed tomography (CT) hypodensities (7), the black hole sign (8), the blend sign (9), and the island sign (10). However, there is an urgent need for further research to identify the prognostic value of biomarkers for HE.

Previous studies have shown that an acute inflammatory reaction may occur within minutes of an $\mathrm{ICH}$, thus triggering an inflammatory cascade that exacerbates secondary brain injury (11). Levels of high-sensitivity C-reactive protein (hs-CRP), as an indicator of inflammation, tend to be higher following stroke. Studies have demonstrated that levels of hs-CRP are also associated with hematoma volume and clinical outcomes after ICH (12). However, the precise relationships between hs-CRP levels, the presence of a spot sign, and HE following spontaneous ICH have yet to be elucidated.

This study aimed to investigate the relationships between hsCRP levels, the presence of a spot sign, HE, and poor clinical outcomes in a cohort of patients suffering from acute ICH.

\section{MATERIALS AND METHODS \\ Study Population}

This study was based on data obtained from a cohort study conducted at 13 hospitals in Beijing, China. The cohort study protocol was approved by the Institutional Review Board (IRB) of Beijing Tiantan Hospital, Capital Medical University, Beijing, China. The ethics committee approved consent by proxy in the ethics statement. Additional ethics approval was required in some of the participating hospitals, and additional consent was obtained if required by the ethics board of specific sites. All patients who had the capacity to understand and sign the documentation provided to them, or their legally authorized representative, were required to provide written and informed consent in a manner that depended on the requirements of the ethics committee at each specific study site. Participating centers collected data and submitted it online to the coordinating center at Beijing Tiantan Hospital, Capital Medical University.

Between December 2014 and September 2016, we recruited 1,964 patients, aged $\geq 18$ years, who had been diagnosed with acute symptomatic ICH by computed tomography. Next, we selected 92 patients from this cohort for the present study (Figure 1). Our inclusion criteria were as follows: patients who presented within $24 \mathrm{~h}$ of the onset of symptoms, data relating to the initial NCCT were available, patients who underwent followup NCCT at $48 \mathrm{~h}$ and multidetector CT angiography (MDCTA) within $48 \mathrm{~h}$, and hs-CRP data were collected at admission. Patients were excluded if they had a form of secondary ICH (hemorrhages resulting from aneurysm, vascular malformation, hemorrhagic infarction, tumors, or impaired coagulation), a history of acute or chronic infection, or underwent surgery prior to the follow-up NCCT and MDCTA.

\section{Collection of Clinical Information}

Demographic and baseline characteristics were collected at admission, including age, gender, alcohol and tobacco use, history of hypertension/diabetes/stroke, concomitant medications (such as antihypertensive/antiplatelet medications), systolic blood pressure (SBP), and routine laboratory data (leukocyte count, platelet count, blood glucose, fibrinogen, activated partial thromboplastin time, and international normalized ratio). We also determined scores for the Glasgow Coma Scale (GCS) and the National Institutes of Health Stroke Scale (NIHSS) at the time of admission. At hospital discharge, antiplatelet medication, antihypertensive medication, in-hospital infections (lung/urinary tract), necessity of operation, and rehabilitation information were collected.

\section{Patient Follow-Up and Clinical Outcomes After ICH}

One year after the indexed ICH events, we carried out telephone interviews to all of the patients to acquire a range of followup data related to functional outcomes, including the modified Rankin Scale (mRS) and the non-fatal recurrence of stroke. Weekly phone calls were carried out until three missed calls were recorded; at this point, patients were classified as being lost to follow-up. A structured interview protocol was used in all telephone follow-ups, and all interviews were carried out by trained personnel. An unfavorable clinical outcome was defined as an mRS score $>2$ after 1 year of follow-up (13).

\section{Radiological Information}

The CT scan protocol was consistent with previous studies (14). In brief, an initial NCCT was performed, using standard clinical parameters (with an axial section thickness of $5 \mathrm{~mm}$ ), on each patient who presented within $24 \mathrm{~h}$ of the onset of symptoms. A follow-up NCCT scan was also performed within $48 \mathrm{~h}$ of admission. A MDCTA scan was also performed to identify spot signs. MDCTA scans were performed with a bolus-tracking method by injecting $90 \mathrm{ml}$ of non-ionic iodinated contrast (OPTIRAY 350) at a rate of $5 \mathrm{ml} / \mathrm{s}$. Data arising from the MDCTA scan were then processed by a CT technologist. All images were viewed in a double-blinded manner on PACS workstations by one neuroradiologist and one neurologist who had no access 
1872 Excluded

301 patients presented without $24 \mathrm{~h}$ of symptom onset

176 patients didn't take initial NCCT within $24 \mathrm{~h}$

999 patients didn't take follow-up NCCT within $48 \mathrm{~h}$

282 patients didn't take MDCTA within 48h

114 patients didn 't measure hs-CRP at admission

1964 Patients with acute symptomatic

ICH (Dec 2014 to Sep 2016)

92 Patients Included

FIGURE 1 | Flow chart of study patients.

to clinical data. These members of clinical staff were asked to identify spot signs in accordance with previously published criteria (15) and to exclude cases involving secondary ICH, such as those caused by aneurysms, vascular malformation, or venous sinus thrombosis. The two clinical staff had to agree on each case; differences in opinion were settled by consensus. We also recorded the time from symptom onset to initial NCCT, the location of the hematoma (supratentorial deep gray matter vs. other locations, such as lobar and infratentorial locations), and the presence of intraventricular hemorrhage (IVH). ICH volumes were determined by manually drawing a hematoma outline on the Siemens multidetector-row scanner using specialized software. HE was diagnosed when there was an increase in hematoma volume by $>33 \%$ or $>12.5 \mathrm{ml}$ (16).

\section{Measurement and Classification of High-Sensitivity CRP}

Blood samples were collected from each patient at admission in EDTA tubes (for plasma) or vacutainer tubes (for serum). Levels of hs-CRP were determined by a particle-enhanced immunoturbidimetric assay (Ultrasensitive CRP kit; Orion Diagnostica, Espoo, Finland) and an auto-analyzer (Hitachi 911; Hitachi, Tokyo, Japan) in the laboratory at Beijing Tiantan Hospital. Levels of hs-CRP level were categorized into two groups according to the laboratory cut-off value: hs-CRP $<3 \mathrm{mg} / \mathrm{L}$ and hs-CRP $\geq 3 \mathrm{mg} / \mathrm{L}$.

\section{Statistical Analysis}

Statistical analyses were performed using the SPSS statistical package version 23.0 (SPSS Inc., Chicago, USA). Continuous variables are described as means \pm standard deviation (SD) or as medians (with interquartile range, IQR) as appropriate. These data were compared using Student $t$-tests or Wilcoxon ranksum tests, accordingly. Categorical variables are presented as numbers (percentages) and were compared using chi-squared tests. Multivariable logistic regression models were carried out using the stepwise-forward method (Wald) and used to calculate odds ratios (ORs) for the relationship between hs-CRP and the risks of spot sign, HE, and unfavorable outcomes 1 year after acute ICH. Variables that were known to be associated with spot sign, $\mathrm{HE}$, or clinical outcomes 1 year after ICH were then included in a multivariate model. Stepwise regression used the SPSS default $p$-value of 0.05 . Multicollinearity analysis indicated that the variance inflation factor (VIF) was $<2.0$ in the parsimonious model. All statistical analyses were two-tailed, and a $p$-value of 0.05 was considered to be statistically significant.

\section{RESULTS}

\section{Baseline Characteristics}

We recruited 92 patients in this study; 54 of these patients had an hs-CRP level $\geq 3 \mathrm{mg} / \mathrm{L}$. The baseline characteristics for all patients and groups according to hs-CRP levels are shown 
TABLE 1 | Baseline characteristics of all patients and patients grouped by hs-CRP levels.

\begin{tabular}{|c|c|c|c|c|}
\hline & \multirow[b]{2}{*}{ All $(n=92)$} & \multicolumn{3}{|c|}{ hs-CRP } \\
\hline & & $<3 \mathrm{mg} / \mathrm{L}(\mathrm{n}=38)$ & $\geq 3 \mathrm{mg} / \mathrm{L}(\mathrm{n}=54)$ & $p$-value \\
\hline Male gender & $64(69.6)$ & $21(55.3)$ & $43(79.6)$ & 0.01 \\
\hline Age, year & $53.0(42.5,60.0)$ & $51.5(41.8,57.3)$ & $56.0(45.5,62.0)$ & 0.16 \\
\hline History of hypertension & $58(63.0)$ & $21(55.3)$ & 37 (68.5) & 0.16 \\
\hline History of diabetes & $8(8.7)$ & $3(7.9)$ & $5(9.3)$ & 0.82 \\
\hline History of stroke & $8(8.7)$ & $4(10.5)$ & $4(7.4)$ & 0.61 \\
\hline Current smoking & $36(39.1)$ & $13(34.2)$ & $23(42.6)$ & 0.42 \\
\hline Alcohol & $46(50.0)$ & $18(47.4)$ & 28 (51.9) & 0.65 \\
\hline Antiplatelet medication & $7(7.6)$ & $2(5.3)$ & $5(9.3)$ & 0.92 \\
\hline Antihypertensive medication & $23(25.0)$ & $10(26.3)$ & $13(24.1)$ & 0.70 \\
\hline Infections (lung/urinary tract) & $29(31.5)$ & $4(10.5)$ & 25 (46.3) & $<0.01$ \\
\hline Necessity of operation & $25(27.2)$ & $3(7.9)$ & $22(40.7)$ & $<0.01$ \\
\hline Rehabilitation & $58(63.0)$ & $23(60.5)$ & $35(64.8)$ & 0.68 \\
\hline GCS score & $14.0(11.3,15.0)$ & $14.0(12.8,15.0)$ & $13.5(10.0,15.0)$ & 0.13 \\
\hline NIHSS score & $11.8 \pm 8.0$ & $9.9 \pm 7.2$ & $13.2 \pm 8.3$ & 0.05 \\
\hline SBP, $\mathrm{mmHg}$ & $163.5 \pm 27.0$ & $161.7 \pm 28.7$ & $164.8 \pm 25.9$ & 0.60 \\
\hline Time from onset to initial NCCT (h) & $4.1(2.3,7.6)$ & $4.6(2.2,7.2)$ & $3.9(2.4,7.9)$ & 0.90 \\
\hline Interval between initial NCCT and follow-up NCCT (h) & $18.5(12.0,24.0)$ & $19.0(12.0,25.0)$ & $18.0(13.0,23.0)$ & 0.58 \\
\hline Baseline hematoma volume (ml) & $22.4(10.0,48.4)$ & $14.5(7.9,27.1)$ & $37.3(14.9,55.4)$ & $<0.01$ \\
\hline Hematoma volume at follow-up (ml) & $25.9(11.0,57.0)$ & $14.4(8.0,28.5)$ & $39.8(18.8,67.4)$ & $<0.01$ \\
\hline Hematoma location & & & & 0.43 \\
\hline Lobar & $35(38.0)$ & $15(39.5)$ & $20(37.0)$ & \\
\hline Deep localization & $54(58.7)$ & $23(60.5)$ & $31(57.4)$ & \\
\hline Infratentorial location & $2(2.2)$ & 0 & $2(3.7)$ & \\
\hline $\mathrm{IVH}$ & $1(1.1)$ & 0 & $1(1.9)$ & \\
\hline Spot sign & $29(31.5)$ & $12(31.6)$ & $17(31.5)$ & 0.99 \\
\hline Hematoma expansion & $27(29.3)$ & $7(18.4)$ & $20(37.0)$ & 0.05 \\
\hline mRS 3-6 at 3 months & $43(46.7)$ & $8(21.1)$ & $35(64.8)$ & $<0.01$ \\
\hline mRS 3-6 at 1 year & $33(35.9)$ & $3(7.9)$ & $30(55.6)$ & $<0.01$ \\
\hline Non-fatal recurrence of stroke at 1 year & $4(4.3)$ & $2(5.3)$ & $2(3.7)$ & 0.80 \\
\hline
\end{tabular}

Data are expressed as $n(\%)$, mean \pm standard deviation (SD), or median (IQR), as appropriate.

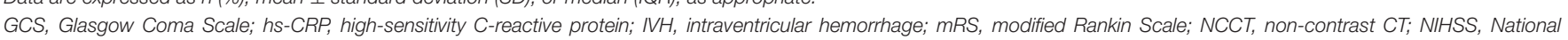
Institutes of Health Stroke Scale; SBP, systolic blood pressure.

in Table 1. The median GCS score for the entire cohort was $14.0(11.3,15.0)$, and the mean NIHSS score was $11.8 \pm 8.0$. The mean duration of time between the onset of symptoms and the initial NCCT was $4.1 \mathrm{~h}$, whereas the median hematoma volumes at baseline and follow-up were $22.4 \mathrm{ml}(10.0,48.4 \mathrm{ml})$ and $25.9 \mathrm{ml}(11.0,57.0 \mathrm{ml})$, respectively. Most of the hematoma was located in the supratentorial deep gray matter (54 patients, $58.7 \%$ of the cohort), followed by lobar locations (35 patients, $38.0 \%$ of the cohort), and infratentorial locations (2 patients, $2.2 \%$ of the cohort). Spot signs and HE were observed in 29 patients $(31.5 \%$ of the cohort) and 27 patients $(29.3 \%$ of the cohort), respectively. Thirty-three patients (35.9\% of the cohort) had poor clinical outcomes 1 year after ICH. Patients with higher hs-CRP levels at admission were more likely to be male and have a larger hematoma volume at baseline and follow-up; these patients also tended to be more prone to infections and surgical interventions. These patients were also more likely to suffer from $\mathrm{HE}$ and poor outcomes 1 year after $\mathrm{ICH}$. Five patients died within 1 year of ICH; all five of these patients had hs-CRP levels $\geq 3 \mathrm{mg} / \mathrm{L}$.

\section{Spot Signs, Radiological Characteristics, and Marker Levels}

Spot signs were detected in 29 out of the 92 recruited patients $(31.5 \%)$. Patients with spot signs were more likely to have a larger hematoma volume at baseline and follow-up (baseline: 44.1 [18.8, $55.2 \mathrm{ml}]$ vs. $16.2 \mathrm{ml}[8.1,38.2 \mathrm{ml}$ ], $p=0.01$; follow-up: 44.4 [23.2, $67.2 \mathrm{ml}]$ vs. $21.0 \mathrm{ml}[8.7,43.9 \mathrm{ml}], p=0.02$ ). However, spot signs were more likely to occur in lobar locations, but less likely to occur in deep locations. Levels of hs-CRP were not significantly associated with spot signs (Table 2).

\section{Correlations Between hs-CRP, Spot Signs, and Hematoma Extension}

Several variables were applied in the stepwise-forward logistic regression for spot signs, including gender, age, baseline 
TABLE 2 | Radiological characteristics and marker levels according to spot sign.

\begin{tabular}{lccc}
\hline Radiological data & $\begin{array}{c}\text { No spot sign } \\
(\boldsymbol{n}=\mathbf{6 3})\end{array}$ & $\begin{array}{c}\text { Spot sign } \\
(\boldsymbol{n}=\mathbf{2 9})\end{array}$ & -value \\
\hline Hematoma location & & & 0.04 \\
$\quad$ Lobar & $20(31.7)$ & $15(51.7)$ & \\
Deep localization & $40(63.5)$ & $14(48.3)$ & \\
IVH & $2(3.2)$ & 0 & \\
Infratentorial location & $1(1.6)$ & 0 & \\
Baseline hematoma volume (ml) & $16.2(8.1,38.2)$ & $44.1(18.8,55.2)$ & 0.01 \\
Hematoma volume at follow-up (ml) & $21.0(8.7,43.9)$ & $44.4(23.2,67.2)$ & 0.02 \\
Hematoma expansion & $20(31.7)$ & $7(24.1)$ & 0.46 \\
Time from onset to initial NCCT (h) & $4.0(2.3,6.4)$ & $4.4(2.3,9.4)$ & 0.59 \\
hs-CRP, mg/L & $6.0(1.8,11.3)$ & $4.0(1.3,11.9)$ & 0.91
\end{tabular}

Data are expressed as $n$ (\%), mean \pm standard deviation $(S D)$, or median (IQR), as appropriate.

hs-CRP, high-sensitivity C-reactive protein; IVH, intraventricular hemorrhage; NCCT, noncontrast CT.

hematoma volume, hematoma location, GCS score, NIHSS score, time from onset to initial NCCT, SBP, antiplatelet medication, and hs-CRP level. These analyses showed that only hematoma location created a good model. There were no useful indicators identified when all the other variables, as well as the incidence of spot signs, were included in stepwise-forward logistic regression for HE. In other words, an hs-CRP level $\geq 3 \mathrm{mg} / \mathrm{L}$ was not a significant indicator for either spot signs $(p=0.68)$ or HE $(p=0.07)$. However, hematoma location (OR: 3.08, 95\% confidence interval [CI]: $1.31-7.22, p=0.01$ ) was identified as an independent predictor for spot signs.

\section{Associations Between hs-CRP Levels and Clinical Outcome}

Next, all variables, including gender, sex, baseline hematoma volume, hematoma location, time from onset to initial NCCT, GCS scores, NIHSS scores at baseline, spot signs, HE, SBP, and hs-CRP levels, were applied into a stepwise-forward logistic regression for the clinical outcome 1 year after ICH. The best models included an hs-CRP level $\geq 3 \mathrm{mg} / \mathrm{L}$, age, baseline hematoma volume, NIHSS score, and HE. Using these four selected indices, we then carried out multicollinearity analysis; the VIF of each of these parameters was $<2.0$. Thus, an hsCRP level $\geq 3 \mathrm{mg} / \mathrm{L}$ (OR: 37.16, 95\% CI: 2.78-496.46, $p=$ 0.006 ), age (OR: $1.25,95 \% \mathrm{CI}: 1.08-1.45, p=0.003)$, baseline hematoma volume (OR: 1.07, 95\% CI: $1.01-1.13, p=0.03$ ), and HE (OR: 18.12, 95\% CI: 1.41-233.32, $p=0.03$ ) were identified as significant and independent predictors for an unfavorable outcome 1 year after acute ICH (Table 3 ).

\section{DISCUSSION}

In this study, we found that an hs-CRP level $\geq 3 \mathrm{mg} / \mathrm{L}$ was a significant indicator of unfavorable outcomes 1 year after ICH, but not an independent risk factor for either spot signs or HE.

Several other factors are worth noting. First, it has been shown that hematoma volume at admission is associated with poor clinical outcomes (17). In the present study, the stepwise-forward
TABLE 3 | Stepwise-forward (Wald) logistic regression for 1-year clinical outcomes stratified by hs-CRP.

\begin{tabular}{|c|c|c|c|}
\hline & \multicolumn{2}{|l|}{$\begin{array}{l}\text { Logistic } \\
\text { regression }\end{array}$} & \multirow{2}{*}{$\begin{array}{c}\text { Collinearity } \\
\text { statistics }\end{array}$} \\
\hline & OR $(95 \% \mathrm{Cl})$ & $p$-value & \\
\hline hs-CRP ( $\geq 3$ mg/L) & $37.16(2.78,496.46)$ & 0.006 & 1.23 \\
\hline Age & $1.25(1.08,1.45)$ & 0.003 & 1.05 \\
\hline Baseline hematoma volume, $\mathrm{ml}$ & $1.07(1.01,1.13)$ & 0.03 & 1.16 \\
\hline NIHSS score & $1.14(0.99,1.31)$ & 0.07 & 1.10 \\
\hline HE & $18.12(1.41,233.32)$ & 0.03 & 1.11 \\
\hline
\end{tabular}

HE, hematoma enlargement; hs-CRP, high-sensitivity C-reactive protein; NIHSS, National Institutes of Health Stroke Scale; VIF, variance inflation factor.

Data were adjusted for age, gender, baseline hematoma volume, hematoma location, time from onset to initial NCCT, GCS score, NIHSS score at baseline, spot sign, SBP, HE, and $h s-C R P$.

logistic regression for clinical outcome 1 year after acute $\mathrm{ICH}$ also showed that baseline hematoma volume was an independent predictor of unfavorable outcome 1 year after ICH. We also found that the baseline hematoma volume in patients with an hs-CRP level $\geq 3 \mathrm{mg} / \mathrm{L}$ (median: $37.3 \mathrm{ml}$ ) was over 2 -fold higher than those in patients with an hs-CRP level $<3 \mathrm{mg} / \mathrm{L}$ (median: $14.5 \mathrm{ml}$ ); the same trend was also evident for hematoma volume at follow-up (median: 39.8 vs. $14.4 \mathrm{ml}$ ). Thus, we conclude that hs-CRP is a good indicator for hematoma volume. We also found that hs-CRP level remained as an independent predictor for poor clinical outcome even after the $\mathrm{ICH}$ volume and $\mathrm{HE}$ were controlled in logistic regression, thus suggesting that hsCRP may also represent a useful marker for further secondary injuries caused by ICH.

Previous research has reported that elevated hs-CRP levels are associated with poor outcomes in several cardiovascular diseases, including ischemic stroke $(18,19)$, subarachnoid hemorrhage $(20,21)$, and acute coronary artery diseases (22). Our present study showed that elevated hs-CRP levels were correlated with poor outcomes in patients suffering from ICH. All of these poor outcomes may arise from the acute inflammatory response that can occur several minutes after ICH and then trigger systemic inflammation $(11,23)$. hs-CRP is an established laboratory marker for inflammation and poor clinical outcomes after ICH (24). However, whether hs-CRP is related to spot signs or HE has not been ascertained in previous research. Our present study showed that elevated hs-CRP levels were associated with poor clinical outcomes, as reported by previous studies, but were not associated with spot signs or HE. The mechanisms underlying the association between hs-CRP levels and poor clinical outcomes have not been fully elucidated, although an inflammatory cascade is believed to play an important role in this process. hs-CRP may cause direct disruption of the blood-brain barrier (BBB) and induce endothelial dysfunction (25); collectively, these processes may result in secondary brain injury, thus leading to poor clinical outcomes (12) and HE. Previous research demonstrated that the peak hs-CRP concentration occurs mainly within $48 \mathrm{~h}$ of acute $\mathrm{ICH}$, and that the hs-CRP level at $24 \mathrm{~h}$ is a more accurate predictor of poor outcomes than at admission (26). In our present study, we only collected blood samples at admission; it is possible 
that this practice may have interfered with our investigations of the association between hs-CRP, spot signs, and HE.

Previous research showed that the spot sign is an independent risk factor for $\mathrm{HE}$ and poor clinical outcomes (27-30), with a sensitivity of $62 \%$ and a specificity of $88 \%$ (30). Previous studies also reported that the incidence of spot sign ranged from 19 to $46 \%$, despite different time intervals between the onset of symptoms and initial imaging $(15,29,31)$. In the present study, we detected the spot sign in $31.5 \%$ of our patient cohort; this incidence was similar to that reported previously. It is thought that the spot sign represents the site of vessel rupture and active bleeding during the early stages, but represents the site of physiological hemostasis, associated microaneurysms, or aneurysm-like lesions during subsequent stages $(32,33)$. It is also evident that "spot sign" and "contrast extravasation" are often misunderstood and considered to be the same. The "spot sign" refers to enhanced foci $\geq 1 \mathrm{~mm}$ in size within a hematoma on CTA images, whereas "contrast extravasation" refers to an increase in contrast density on enhanced CT images (33). Therefore, we used the term "spot sign" in our current study as our analysis included CTA images. A number of risk factors have been associated with spot signs, including anticoagulation, the apolipoprotein E\&2 allele, a larger initial hematoma volume, early presentation, lower GCS scores at admission, a mean arterial blood pressure $>120 \mathrm{mmHg}$, and the presence of IVH (34). HE often occurs within $4 \mathrm{~h}$ of the onset of symptoms, although in some cases, HE occurs within the subsequent $21 \mathrm{~h}$ (35). The risk factors for $\mathrm{HE}$ are similar to those for spot signs (35). At present, there are a number of different criteria used to diagnose HE; in the present study, we used the most widely accepted criteria (35). We found that HE is a risk factor for unfavorable outcomes 1 year after ICH; spot sign was not a risk factor for an unfavorable outcome. Overall, our data suggest that elevated hs-CRP levels in the early stages of ICH may be associated with poor outcomes. Our findings may have some potential clinical applications. For example, future clinical trials designed to test hemostatic agents and neuroprotective drugs should include hs-CRP levels as an efficient biomarker. Large-scale studies are now needed to verify our current findings.

This study has several limitations that need to be considered. First, the data used in our study were obtained from an existing database and not collected prospectively from consecutive patients. This practice may have resulted in potential bias, such as selection bias, as patients with higher NIHSS scores and larger hematomas may not have been able to undergo followup CT scans within $48 \mathrm{~h}$. Thus, the presence of spot signs and HE could have been underestimated. Second, almost onethird of the patients in our study underwent neurosurgery $48 \mathrm{~h}$ after the onset of symptoms; this may have influenced our analysis of clinical outcomes 1 year after ICH. However, the Surgical Trial in Intracerebral Hemorrhage (STICH) trial $(36,37)$ and the STICH II trial $(38)$ showed that there were no significant differences in functional outcomes between a surgery group and a medical group of patients with spontaneous supratentorial lobar intracerebral hematomas. Moreover, the minimally invasive surgery with thrombolysis in intracerebral hemorrhage evacuation (MISTIE III) trial showed that the minimally invasive surgical evacuation of $\mathrm{ICH}$ did not improve the clinical outcomes 1 year after ICH when compared with nonsurgical medical care (37). Further studies are now needed to verify these findings. Third, we recruited patients who presented within $24 \mathrm{~h}$ of the onset of symptoms; MDCTA was usually performed within $48 \mathrm{~h}$ and reported in a previous study (26); this practice could have also led to the underestimation of HE. Nevertheless, the incidence of HE in our study was similar to that reported in other studies. Finally, our study cohort only included 92 patients and may, therefore, have had limited statistical power. Further research now needs to investigate the prognostic value of biomarkers for spot signs and HE.

\section{CONCLUSION}

In conclusion, our study demonstrated that an hs-CRP level $\geq 3 \mathrm{mg} / \mathrm{L}$ was a significant indicator of unfavorable clinical outcomes 1 year after acute ICH. However, in this study, we only selected 92 patients from a cohort of 1,872 patients, which may lead to selection bias with $<5 \%$ of patients. Further prospective studies, with large sample sizes, are now needed to identify the mechanisms underlying this relationship.

\section{DATA AVAILABILITY STATEMENT}

The datasets generated for this study are available on request to the corresponding author.

\section{ETHICS STATEMENT}

The studies involving human participants were reviewed and approved by the Institutional Review Board (IRB) of the Beijing Tiantan Hospital, Capital Medical University. The patients/participants provided their written informed consent to participate in this study.

\section{AUTHOR CONTRIBUTIONS}

JW conducted the statistical analyses and drafted the original manuscript. WW interpreted the data and conceptualized the study. YL performed the imaging assessment. XZ researched the literature and designed the study.

\section{FUNDING}

This research was supported by grants from the National Key R\&D Program of China (2017YFC1310902), the National Science and Technology Major Project (2017ZX09304018), and the Beijing Municipal Administration of Hospitals' Youth Project (QML20150503).

\section{ACKNOWLEDGMENTS}

We would like to thank the centers and all the members who participated in this study. 


\section{REFERENCES}

1. Rajashekar D, Liang JW. Intracerebral Hemorrhage. Treasure Island, FL: StatPearls Publishing StatPearls Publishing LLC (2020).

2. Dowlatshahi D, Brouwers HB, Demchuk AM, Hill MD, Aviv RI, Ufholz LA, et al. Predicting intracerebral hemorrhage growth with the spot sign: the effect of onset-to-scan time. Stroke. (2016) 47:695-700. doi: 10.1161/STROKEAHA.115.012012

3. Ziai WC, Carhuapoma JR. Intracerebral hemorrhage. Continuum. (2018) 24:1603-22. doi: 10.1212/CON.0000000000000672

4. Nawabi J, Elsayed S, Kniep H, Sporns P. Inter- and intrarater agreement of spot sign and noncontrast CT markers for early intracerebral hemorrhage expansion. J Clin Med. (2020) 9:1020. doi: 10.3390/jcm 90 41020

5. Sprigg N, Flaherty K, Appleton JP, Al-Shahi Salman R, Bereczki $\mathrm{D}$, Beridze $\mathrm{M}$, et al. Tranexamic acid for hyperacute primary IntraCerebral Haemorrhage (TICH-2): an international randomised, placebo-controlled, phase 3 superiority trial. Lancet. (2018) 391:2107-15. doi: 10.1016/S0140-6736(18)31033-X

6. Morotti A, Brouwers HB, Romero JM, Jessel MJ, Vashkevich A, Schwab $\mathrm{K}$, et al. Intensive blood pressure reduction and spot sign in intracerebral hemorrhage: a secondary analysis of a randomized clinical trial. JAMA Neurol. (2017) 74:950-60. doi: 10.1001/jamaneurol.2017.1014

7. Boulouis G, Morotti A, Brouwers HB, Charidimou A, Jessel MJ, Auriel E, et al. Association between hypodensities detected by computed tomography and hematoma expansion in patients with intracerebral hemorrhage. JAMA Neurol. (2016) 73:961-8. doi: 10.1001/jamaneurol. 2016.1218

8. Li Q, Zhang G, Xiong X, Wang XC, Yang WS, Li KW, et al. Black hole sign: novel imaging marker that predicts hematoma growth in patients with intracerebral hemorrhage. Stroke. (2016) 47:1777-81. doi: 10.1161/STROKEAHA.116.013186

9. Li Q, Zhang G, Huang YJ, Dong MX, Lv FJ, Wei X, et al. Blend sign on computed tomography: novel and reliable predictor for early hematoma growth in patients with intracerebral hemorrhage. Stroke. (2015) 46:211923. doi: 10.1161/STROKEAHA.115.009185

10. Li Q, Liu QJ, Yang WS, Wang XC, Zhao LB, Xiong X, et al. Island sign: an imaging predictor for early hematoma expansion and poor outcome in patients with intracerebral hemorrhage. Stroke. (2017) 48:301925. doi: 10.1161/STROKEAHA.117.017985

11. Shi K, Tian DC, Li ZG, Ducruet AF, Lawton MT, Shi FD. Global brain inflammation in stroke. Lancet Neurol. (2019) 18:1058-66. doi: 10.1016/S1474-4422(19)30078-X

12. Di Napoli M, Parry-Jones AR, Smith CJ, Hopkins SJ, Slevin M, Masotti $\mathrm{L}$, et al. C-reactive protein predicts hematoma growth in intracerebral hemorrhage. Stroke. (2014) 45:59-65. doi: 10.1161/STROKEAHA.113. 001721

13. Ji R, Shen H, Pan Y, Wang P, Liu G, Wang Y, et al. A novel risk score to predict 1-year functional outcome after intracerebral hemorrhage and comparison with existing scores. Crit Care. (2013) 17:R275. doi: 10.1186/ cc13130

14. Li N, Wang Y, Wang W, Ma L, Xue J, Weissenborn K, et al. Contrast extravasation on computed tomography angiography predicts clinical outcome in primary intracerebral hemorrhage: a prospective study of 139 cases. Stroke. (2011) 42:3441-6. doi: 10.1161/STROKEAHA.111. 623405

15. Delgado Almandoz JE, Yoo AJ, Stone MJ, Schaefer PW, Goldstein JN, Rosand J, et al. Systematic characterization of the computed tomography angiography spot sign in primary intracerebral hemorrhage identifies patients at highest risk for hematoma expansion: the spot sign score. Stroke. (2009) 40:29943000. doi: 10.1161/STROKEAHA.109.554667

16. Broderick JP, Diringer MN, Hill MD, Brun NC, Mayer SA, Steiner T, et al. Determinants of intracerebral hemorrhage growth: an exploratory analysis. Stroke. (2007) 38:1072-5. doi: 10.1161/01.STR.0000258078.35316.30

17. Hemphill JC, 3rd, Bonovich DC, Besmertis L, Manley GT, Johnston SC. The ICH score: a simple, reliable grading scale for intracerebral hemorrhage. Stroke. (2001) 32:891-7. doi: 10.1161/01.STR.32.4.891
18. Vangilder RL, Davidov DM, Stinehart KR, Huber JD, Turner RC, Wilson $\mathrm{KS}$, et al. C-reactive protein and long-term ischemic stroke prognosis. J Clin Neurosci. (2014) 21:547-53. doi: 10.1016/j.jocn.2013.06.015

19. Di Napoli M, Papa F, Bocola V. C-Reactive protein in ischemic stroke : an independent prognostic factor. Stroke. (2001) 32:917-24. doi: 10.1161/01.STR.32.4.917

20. Lee S, Kim YO, Ryu JA. Clinical usefulness of early serial measurements of C-reactive protein as outcome predictors in patients with subarachnoid hemorrhage. BMC Neurol. (2020) 20:112. doi: 10.1186/s12883-020-01687-3

21. Fountas KN, Tasiou A, Kapsalaki EZ, Paterakis KN, Grigorian AA, Lee GP, et al. Serum and cerebrospinal fluid C-reactive protein levels as predictors of vasospasm in aneurysmal subarachnoid hemorrhage. clinical article. Neurosurg Focus. (2009) 26:E22. doi: 10.3171/2009.2.FOCUS08311

22. Berk BC, Weintraub WS, Alexander RW. Elevation of C-reactive protein in "active" coronary artery disease. Am J Cardiol. (1990) 65:16872. doi: 10.1016/0002-9149(90)90079-G

23. Zhou Y, Wang Y, Wang J, Anne Stetler R, Yang QW. Inflammation in intracerebral hemorrhage: from mechanisms to clinical translation. Prog Neurobiol. (2014) 115:25-44. doi: 10.1016/j.pneurobio.2013.11.003

24. Bernstein JE, Savla P, Dong F, Zampella B, Wiginton JGT., Miulli DE, et al. Inflammatory markers and severity of intracerebral hemorrhage. Cureus. (2018) 10:e3529. doi: 10.7759/cureus.3529

25. Di Napoli M, Slevin M, Popa-Wagner A, Singh P, Lattanzi S, Divani AA. Monomeric C-reactive protein and cerebral hemorrhage: from bench to bedside. Front Immunol. (2018) 9:1921. doi: 10.3389/fimmu.2018.01921

26. Di Napoli M, Godoy DA, Campi V, Del Valle M, Pinero G, Mirofsky M, et al. C-reactive protein level measurement improves mortality prediction when added to the spontaneous intracerebral hemorrhage score. Stroke. (2011) 42:1230-6. doi: 10.1161/STROKEAHA.110.604983

27. Malik R, Chauhan G, Traylor M, Sargurupremraj M, Okada Y, Mishra A, et al. Multiancestry genome-wide association study of 520,000 subjects identifies 32 loci associated with stroke and stroke subtypes. Nat Genet. (2018) 50:524-37.

28. Demchuk AM, Dowlatshahi D, Rodriguez-Luna D, Molina CA, Blas YS, Dzialowski I, et al. Prediction of haematoma growth and outcome in patients with intracerebral haemorrhage using the CT-angiography spot sign (PREDICT): a prospective observational study. Lancet Neurol. (2012) 11:30714. doi: 10.1016/S1474-4422(12)70038-8

29. Phan TG, Krishnadas N, Lai VWY., Batt M, Slater LA, Chandra $\mathrm{RV}$, et al. Meta-analysis of accuracy of the spot sign for predicting hematoma growth and clinical outcomes. Stroke. (2019) 50:2030-6. doi: 10.1161/STROKEAHA.118.024347

30. Xu X, Zhang J, Yang K, Wang Q, Xu B, Chen X. Accuracy of spot sign in predicting hematoma expansion and clinical outcome: a meta-analysis. Medicine. (2018) 97:e11945. doi: 10.1097/MD.0000000000011945

31. Wang B, Yan S, Xu M, Zhang S, Liu K, Hu H, et al. Timing of occurrence is the most important characteristic of spot sign. Stroke. (2016) 47:12338. doi: 10.1161/STROKEAHA.116.012697

32. Dowlatshahi D, Wasserman JK, Momoli F, Petrcich W, Stotts G, Hogan M, et al. Evolution of computed tomography angiography spot sign is consistent with a site of active hemorrhage in acute intracerebral hemorrhage. Stroke. (2014) 45:277-80. doi: 10.1161/STROKEAHA.113.003387

33. Wada R, Aviv RI, Fox AJ, Sahlas DJ, Gladstone DJ, Tomlinson $\mathrm{G}$, et al. CT angiography "spot sign" predicts hematoma expansion in acute intracerebral hemorrhage. Stroke. (2007) 38:1257-62. doi: 10.1161/01.STR.0000259633.59404.f3

34. Brouwers HB, Goldstein JN, Romero JM, Rosand J. Clinical applications of the computed tomography angiography spot sign in acute intracerebral hemorrhage: a review. Stroke. (2012) 43:3427-32. doi: 10.1161/STROKEAHA.112.664003

35. Steiner $T$, Bosel J. Options to restrict hematoma expansion after spontaneous intracerebral hemorrhage. Stroke. (2010) 41:402-9. doi: 10.1161/STROKEAHA.109.552919

36. Mendelow AD, Gregson BA, Fernandes HM, Murray GD, Teasdale GM, Hope DT, et al. Early surgery versus initial conservative treatment in patients with spontaneous supratentorial intracerebral haematomas in the International surgical trial in intracerebral Haemorrhage (STICH): a randomised trial. Lancet. (2005) 365:387-97. doi: 10.1016/S0140-6736(05)70233-6 
37. Hanley DF, Thompson RE, Rosenblum M, Yenokyan G, Lane K, Mcbee $\mathrm{N}$, et al. Efficacy and safety of minimally invasive surgery with thrombolysis in intracerebral haemorrhage evacuation (MISTIE III): a randomised, controlled, open-label, blinded endpoint phase 3 trial. Lancet. (2019) 393:1021-32. doi: 10.1016/S0140-6736(19) 30195-3

38. Mendelow AD, Gregson BA, Rowan EN, Murray GD, Gholkar A, Mitchell PM. Early surgery versus initial conservative treatment in patients with spontaneous supratentorial lobar intracerebral haematomas (STICH II): a randomised trial. Lancet. (2013) 382:397-408. doi: 10.1016/S0140-6736(13)60986-1
Conflict of Interest: The authors declare that the research was conducted in the absence of any commercial or financial relationships that could be construed as a potential conflict of interest.

Copyright (c) 2020 Wang, Wang, Liu and Zhao. This is an open-access article distributed under the terms of the Creative Commons Attribution License (CC BY). The use, distribution or reproduction in other forums is permitted, provided the original author(s) and the copyright owner(s) are credited and that the original publication in this journal is cited, in accordance with accepted academic practice. No use, distribution or reproduction is permitted which does not comply with these terms. 